\title{
Plasmapheresis in the Treatment of Neurologic Disorders in Bangladesh: A Review Update
}

\author{
Sharmin Jahan ${ }^{1}$, Mohammad Enayet Hussain², Md Badrul Alam³, \\ Quazi Deen Mohammad ${ }^{4}$
${ }^{1}$ Assistant Professor, Department of Endocrinology, Bangabandhu Sheikh Mujib Medical University, Dhaka, Bangladesh; ${ }^{2}$ Assistant Professor, Department of Neurophysiology, National Institute of Neurosciences \& Hospital, Dhaka, Bangladesh; ${ }^{3}$ Professor, Department of Neurology, National Institute of Neurosciences \& Hospital, Dhaka, Bangladesh; ${ }^{4}$ Professor, Department of Neurology, National Institute of Neurosciences \& Hospital, Dhaka, Bangladesh

[Received: March 2015; Reviewed: May 2015; Accepted: June 2015; Published: July 2015]

\begin{abstract}
Plasmapheresis is a very effective modality of treatment for many of the immune-mediated neurological disorders. It is a relatively new concept in the context of our country. Although it was previously done in one or two of the private hospitals but the expense was beyond the affordability of m59ost of our patients. Since its inception on September, 2012 the National Institute of Neurosciences \& Hospital (NINS) has made some major advancement in patient management. Initiation of plasmapheresis at an affordable cost is one such example. In this article we intend to share our experience of the few cases who have received this treatment. [Journal of National Institute of Neurosciences Bangladesh, 2015;1(2): 62-64]
\end{abstract}

Keywords: Plasmapheresis; neurologic disorders; immune-mediated; IVIg

Correspondence: Dr. Sharmin Jahan, Assistant Professor, Department of Endocrinology, Bangabandhu Sheikh Mujib Medical University, Dhaka, Bangladesh; Email: Sharmindme@yahoo.com

Conflict of interest: There is no conflict of interest.

Funding agency: The study was not funded by any authority.

Contribution to authors: SJ and MEH were involved in manuscript preparation as well as involved in literature search up and MBA \& QDM were involved in manuscript writing and manuscript revision.

How to cite this article: Jahan S, Hussain ME, Alam MB, Mohammad QD. Plasmapheresis in the Treatment of Neurologic Disorders in Bangladesh: A Review Update. J Natl Inst Neurosci Bangladesh, 2015;1(2): 62-64

\section{Introduction}

Plasmapheresis (from the Greek $\pi \lambda \alpha \dot{\sigma} \sigma \alpha$ - plasma,

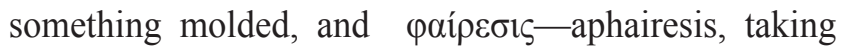
away) is the removal, treatment, and return of (components of) blood plasma from blood circulation'. It is thus an extracorporeal therapy. The most common term is plasmapheresis, which technically means removal of plasma only, for the proper term, "plasma exchange", which technically refers to both removal of plasma and its replacement ${ }^{2}$. In the literature, these terms are used interchangeably.

\section{History}

Michael Rubinstein was the first person to use plasmapheresis to treat an immune-related disorder when he "saved the life of an adolescent boy with thrombotic thrombocytopenic purpura (TTP) at the old
Cedars of Lebanon Hospital in Los Angeles in $1959^{3}$. The modern plasmapheresis process itself originated in the "[U.S.] National Cancer Institute between 1963 and 1968, [where] investigators drew upon an old dairy creamer separation technology first used in 1878 and refined by Edwin Cohn's centrifuge ${ }^{4}$ marketed in 1953.

\section{Mechanics}

During plasmapheresis, blood is initially taken out of the body through a needle or previously implanted catheter ${ }^{5}$. Plasma is then removed from the blood by a cell separator. Three procedures are commonly used to separate the plasma from the blood cells, with each method having its own advantages and disadvantages ${ }^{6}$ :

- Discontinuous flow centrifugation: One venous catheter line is required. Typically, a $300 \mathrm{ml}$ batch of blood is removed at a time and centrifuged to separate 
plasma from blood cells.

- Continuous flow centrifugation: Two venous lines are used. This method requires slightly less blood volume out of the body at any one time, as it is able to continuously spin out plasma.

- Plasma filtration: Two venous lines are used. The plasma is filtered using standard hemodialysis equipment. This continuous process requires that less than $100 \mathrm{ml}$ of blood be outside the body at one time.

After plasma separation, the blood cells are returned to the person undergoing treatment, while the plasma, which contains the antibodies, is first treated and then returned to the patient in traditional plasmapheresis. In plasma exchange, the removed plasma is discarded and the patient receives replacement donor plasma, albumin, or a combination of albumin and saline (usually $70 \%$ albumin and $30 \%$ saline). An anticoagulant usually citrate is given to the patient during the procedure. Plasmapheresis is used as a therapy in particular diseases. It is an uncommon treatment in the United States, but it is more common in Europe and particularly Japan.

\section{Presumed Mechanisms}

A variety of possible mechanisms for the actions of therapeutic PP has been proposed, including removal of antibody, alloantibody, immune complexes, monoclonal protein, toxin or cytokine(s), replenishment of a specific plasma factor, and, lastly, the placebo effect ${ }^{7}$. For most neurologic diseases, patient improvement is associated with a drop in antibody titers as a result of PP. It should be noted, however, that other mechanisms may exist. Indeed, PP can be looked upon as a "blunderbuss" that removes all the nonformed elements in plasma, including immunoglobulin, cytokines, and other serum factors, in a nonspecific fashion ${ }^{8}$. The specific factor whose removal is crucial in therapeutically successful PP is thus not specifically known.

\section{Complications of Plasmapheresis Therapy}

Though plasmapheresis is helpful in certain medical conditions, like any other therapy, there are potential risks and complications. Insertion of a rather large intravenous catheter can lead to bleeding, pneumothorax, thrombosis (in case of CV line), and

Table 1: Indication of Plasmapheresis

\begin{tabular}{|c|c|}
\hline Neurologic & Non-neurologic \\
\hline 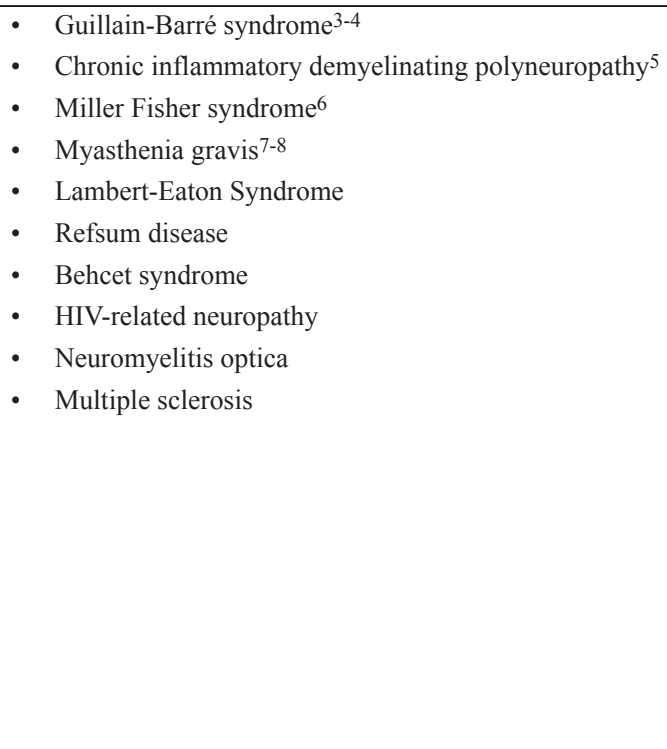 & $\begin{array}{l}\text { - } \text { Graves' disease in infants and neonates } \\
\text { - } \text { Idiopathic pulmonary fibrosis } \\
\text { - } \text { Hoodpasture's syndrome } \\
\text { o Cryoglobulinemia } \\
\text { o Paraproteinemia } \\
\text { - Waldenström macroglobulinemia } \\
\text { - Thrombotic thrombocytopenic purpura (TTP)/hemolytic uremic } \\
\text { - } \text { syndrome } \\
\text { - Antiphospholipid Antibody Syndrome (APS or APLS) } \\
\text { - Microscopic polyangiitis } \\
\text { - Recurrent focal and segmental glomerulosclerosis in the transplanted } \\
\text { - } \text { kidney } \\
\text { - Pemples syndrome } \\
\text { - Rhabdomyolysis } \\
\text { - Toxic Epidermal Necrolysis (TEN) }\end{array}$ \\
\hline
\end{tabular}

thrombophlebitis ${ }^{3}$. Aside from placing the catheter, the procedure itself has complications. Citrate is infused while the blood is running through the circuit. It is an effective anticoagulant that binds to calcium in the blood. This can lead to life-threateningly hypocalcaemia which can be detected using the Chvostek's sign or Trousseau's sign ${ }^{5}$. To prevent this complication, calcium is infused intravenously while the patient is undergoing the plasmapheresis; in addition, calcium supplementation by mouth may also be given. Other complications include ${ }^{8}$ :

- Hypotension

- Potential exposure to blood products, with risk of transfusion reactions or transfusion transmitted diseases

- Suppression of the patient's immune system 
- Bleeding or hematoma from needle placement

\section{Plasmapheresis in Bangladesh}

The procedure was first performed on the $23^{\text {rd }} \mathrm{Feb}$, 2014 at the dept. of transfusion medicine, National Institute of Neurosciences \& Hospital, Dhaka, Bangladesh(NINS). A 45-yr-old male, suffering from
GBS with impending respiratory failure received this treatment and made remarkable recovery. A highly sophisticated, multi-functional Com-TEC plasmapheresis machine imported from Fresenius Kabi AG (Germany) is used for this purpose. The expenditure is relatively less in contrast to that of the private hospitals, Which is around 20,000 taka/cycle.

Table 2: Demographic character, diagnosis \& indication of plasmapheresis in patients

\begin{tabular}{c|c|c|l|l|l}
\hline Case no. & Age & Sex & Diagnosis & Co-morbidities & Indication of plasmapheresis \\
\hline 01. & 39 & M & CIDP & Hypothyroidism & Limitation of ADL (Quadriparesis, bulbar palsy) \\
02. & 44 & M & GBS & IHD, HTN & Limitation of ADL (Quadriparesis) \\
03. & 45 & M & GBS & None & Limitation of ADL (Quadriparesis) \\
04 & 29 & M & GBS & None & Impending res. Failure, bulbar palsy \\
05 & 8 & M & CIDP & DM, HTN & Quadreparesis, sensory impairment \\
06 & 33 & F & GBS & None & Limitation of ADL (Quadreparesis) \\
07 & 55 & M & MG & DM, HTN, IHD & Impending res. failure, bulbar palsy \\
08 & 55 & M & GBS & None & Bulbar palsy \\
\end{tabular}

CIDP-Chronic Inflammatory Demyelinating Neuropathy, GBS- Guillain Barre Syndrome, MG- Myasthenia Gravis, IHD-Ischemic Heart Disease, DM-Diabetes Mellitus, HTN- Hypertension

Generally four to six cycles are required as treatment. The machine uses the continuous flow centrifugation technique- blood at $25 \mathrm{ml} / \mathrm{min}$ is removed \& replaced with reconstituted fluid at $21 \mathrm{ml} / \mathrm{min}$. Patients need some investigation like $\mathrm{CBC} \& \mathrm{Hct}$, viral screening (HBV, HCV, HIV), total plasma protein and albumin, s. electrolyte, plasma calcium, magnesi before undergoing the procedure. During the procedure patients are carefully monitored for their vital parameter as well as any sign of complication. So far 8 patients have received this treatment. Their outcome in term of improvement/deterioration along with any adverse reactions was observed. Here is a brief summary of our observation;

\section{Conclusion}

Plasmapheresis is used for immune-mediated neurological disorders which is limited treatment modalities in Bangladesh. Application of this treatment facility at National Institute of Neurosciences \& Hospital has given a new window in a low cost. This facility should be applied for the neurologic disorders patients of this country.

\section{References}

1. Wallace D J Apharesis for lupus erythematosus. Lupus 1999; 8: 174-180

2. Drew MJ. Plasmapheresis in the dysproteinemias "Therapeutic apheresis". official journal of the International Society for Apheresis and the Japanese Society for Apheresis .2002; 6 (1): 45-52

3. Osterman PO, Lundemo G, Pirskanen R, Fagius J, Pihlstedt P, Siden A. Beneficial effects of plasma exchange in acute inflammatory polyradiculoneuropathy. Lancet 1984;2:1296-1298

4. Guillain-Barre Study Group. Plasmapheresis and acute Guillain-Barre syndrome. Neurology 1985; 35:1096-1104

5. Dyck PJ, Daube J, O'Brien P, et al. Plasma exchange in chronic inflammatory demyelinating polyradiculoneuropathy. N Engl J Med $1986 ; 314: 461-465$

6. Mori M, Kuwabara S, Fukutake T, Hattori T. Plasmapheresis and Miller Fisher syndrome: analysis of 50 consecutive cases. J Neurolog Neurosur Psychiatry 2002;72 (5): 68

7. Batocchi AP, Evoli A, Di-Schino C, Tonali P. Therapeutic apheresis in myasthenia gravis". Therapeutic apheresis : official journal of the International Society for Apheresis and the Japanese Society for Apheresis.2000; 4 (4): 275

8. Dau PC, Lindstrom JM, Cassel CK, Denys EH, Shev EE, Spitler LE. Plasmapheresis and immunosuppressive drug therapy in myasthenia gravis. N Engl J Med 1977;297:1134-1140 